Proyecciones

Vol. $12 \mathrm{~N}^{\circ} 2$, pp.137-147 December 1993

Universidad Católica del Norte

Ant ofagasta - Chile

\title{
ON THE CONTINUITY OF FUZZY INTEGRAL IN RELATION WITH MEASURE CONVERGENCE*
}

\author{
ARTURO FLORES F. and HERIBERTO ROMAN F. \\ Universidad de Tarapacá, Arica, Chile. \\ R.C. BASSANEZI \\ Universidad de Campinas, Campinas, Brasil.
}

\begin{abstract}
The aim of this paper is to analyze the continuity of the fuzzy integral in relation to the measure convergence. More precisely, we introduce the concept of autocontinuity of a fuzzy measure with respect to another fuzzy measure and we establish necessary and sufficient conditions such that measure convergence of functions, with respect to a fuzzy measure, implies the convergence of the fuzzy integral with respect to another fuzzy measure.
\end{abstract}

* This work was partially supported by DIEXA-Universidad de Tarapacá, Proyects $N^{\circ} 4716-91$ and 4731-92. 


\section{Introduction.}

The fuzzy integral is a mathematical tool that has been showed higly efficient with respect to the treatment of problems with no-deterministic nature, like prediction problems or making-decision processes, in presence of diffuse (fuzzy) information. A lot of these problems have many applications in the real world.

That is the reason, moreover others of theoretical order, why this new integral operator has been investigated in the last years about its properties and beliavior.

The nature and genesis of the fuzzy integral are quite different from the classic integral, because while the last one is related with vectorial structure of the basic space, the fuzzy one is related with the lattice structure, in the sense that is calculated by a max-min average. In fact, if $(X, \mathcal{A}, \mu)$ is a fuzzy measure space and $f: X \longrightarrow[0,1]$ is a $\mu$-measurable function, then the fuzzy integral of $f$ can be defined by the expression:

$$
\int f d \mu=\sup _{A \in \mathcal{A}}\left[\mu(A) \wedge \inf _{x \in A} f(x)\right] \quad(\wedge \text { denotes the minimum })
$$

Nevertheless that differences, its surprising how a lot of similarities there exist among them, in the sense that a great number of basic properties of the classic integral (except the vectorials ones) are preserved by the fuzzy integral. In particular, those related to continuity theorems of the integral respect to different kinds of convergence.

In this paper, we introduce the concept of autocontinuity of a fuzzy measure respect to another one and we show the following equivalence:

$$
\mu \text { autocontinuous respect to } \nu \Longleftrightarrow\left(f_{n} \stackrel{\nu}{\longrightarrow} f \Longrightarrow \int f_{n} d \mu \longrightarrow \int f d \mu\right) \text {. }
$$

We also give some examples and applications.

\section{Preliminaries.}

Definition 2.1. Let $X$ be a non empty set and let $\mathcal{A}$ be a $\sigma$-algebra of subsets of $X$. A fuzzy measure on $X$ is an application $\mu: \mathcal{A} \longrightarrow[0,1]$ such that:

F1) $\mu(0)=0, \mu(X)=1$

F2) $A, B \in \mathcal{A}$ and $A \subseteq B \Longrightarrow \mu(A) \leq \mu(B)$ 
Furthermore, if:

F3) $A_{n} \nearrow A \Longrightarrow \mu(A) \nearrow \mu(A)$, then $\mu$ is upper continuous.

Analogously, if:

F4) $A_{n} \searrow A \Longrightarrow \mu\left(A_{n}\right) \searrow \mu(A)$, then $\mu$ is lower continuous.

If $\mu$ satisfies F3) and F4) then we say that $\mu$ is continuous.

The triple $(X, \mathcal{A}, \mu)$ is called a fuzzy measure space.

We denote by $\mathbb{E}(X)=\{f: X \longrightarrow[0,1] / f \mu$ - measurable $\}$.

The fuzzy integral $\int f() d \mu$, in the sense of Sugeno [5], is defined by:

$$
\int f d \mu=\sup _{\alpha \in[0,1]}[\alpha \wedge \mu(A \cap\{f \geq \alpha\}] \quad \forall f \in \mathbb{E}(X), A \in \mathcal{A}
$$

This definition is equivalent to (1). See [3].

Some properties of this integral are:

Theorem 2.2. ([4]).

P1) $\int f d \mu=\int \chi_{A} f d \mu\left(\chi_{A}\right.$ is the characteristic function of $\left.A\right)$

P2) $\int k d \mu=k \wedge \mu(A) \quad k \in[0,1]$ is a constant.

P3) i) If $A \subseteq B$ then $\int_{A} f d \mu \leq \int_{B} f d \mu$

ii) If $f \leq g$ in $A$ then $\int_{A} f d \mu \leq \int_{A} g d \mu$

P4) i) If $\mu(A)=0$ then $\int_{A} f d \mu=0$

ii) If $\int_{A} f d \mu=0$ and $\mu$ is upper continuous then $\mu(A \cap\{f>0\})=0$

P5) $\int_{A}(f+k) d \mu \leq \int_{A} f d \mu+\int_{A} k d \mu, \quad k \in[0,1]$ is a constant.

P6) If $|f-g| \leq \varepsilon$ in $A$, then $\left|\int_{A} f d \mu-\int_{A} g d \mu\right| \leq \varepsilon$.

\section{Remark 2.3.}

i) In the proof of the previous theorem, except by P4), it is not necessary to lave continuity conditions upon $\mu$.

ii) From P1) and P2) we conclude that $\int \chi_{A} d \mu=\mu(A)$. 
iii) From P6) we conclude that the fuzzy integral is continuous respect to uniform convergence.

Definition 2.4. ([6]). Let $(X, \mathcal{A}, \mu)$ a fuzzy measure space. Then:

i) $\boldsymbol{\mu}$ is autocontinuous if:

$\forall A \in \mathcal{A}, \quad\left(B_{n}\right) \subseteq \mathcal{A}$ with $\lim _{n \rightarrow \infty} \mu\left(B_{n}\right)=0$, then $\lim _{n \rightarrow \infty} \mu\left(A \Delta B_{n}\right)=\mu(A)$

( $\Delta$ denote the simetric difference).

ii) $\mu$ is subadditive if $\forall A, B \in \mathcal{A}, \mu(A \cup B) \leq \mu(A)+\mu(B)$.

It is easy to prove that:

Proposition 2.5. Subadditivity $\Longrightarrow$ Autocontinuity.

The definition of convergence in measure for a sequence of measurable functions is analogous to the classic context. Therefore,

$$
f_{n} \longrightarrow f \text { in measure }\left(f_{n} \stackrel{\mu}{\longrightarrow} f\right) \text { if } \forall \varepsilon>0, \lim _{n \rightarrow \infty} \mu\left\{x /\left|f_{n}(x)-f(x)\right| \geq \mathcal{E}\right\}=0 \text {. }
$$

Ralescu and Adams ([3], 1980) proved the next theorem of continuity for the fuzzy integral:

Theroem 2.6. ([3]). Let $\mu$ a continuous fuzzy measure on $X$. If $\mu$ is subadditive and $f_{n} \stackrel{\mu}{\longrightarrow} f$ then $\int f_{n} d \mu \longrightarrow \int f d \mu$.

Wang ([6] 1984) used the concept of autocontinuity for a continuous fuzzy measure and proved the next result:

Theorem 2.7. ([6]), Let $\mu$ a continuous fuzzy measure on $X$. Then:

$$
\left(f_{n} \stackrel{\mu}{\longrightarrow} f \Longrightarrow \int f_{n} d \mu \longrightarrow \int f d \mu\right) \Longleftrightarrow \mu \text { autocontinuous. }
$$

Remark 2.8. In [4], the previous theorem is proved without using the continuity of fuzzy measure $\mu$.

Greco and Bassanezi [2], introduce the concept of F-continuity and, in a recent paper [1], they show the continuity of the fuzzy integral respect to convergence in measure when this is $\mathrm{F}$-continuous, improving partially the Theorem 2.7 . 
Definition 2.9. Let $\mu$ and $\nu$ fuzzy measures on $\Lambda$. We say $i$ i al $\mu$ is $r$-conthuous with respect to $\nu$ (over a $\sigma$-algebra $\mathbf{A}$ ) if:

$$
A, A_{n} \in \mathbf{A} \text { and } \nu\left(A_{n} \Delta A\right) \longrightarrow 0 \text { then } \mu\left(A_{n}\right) \longrightarrow \mu(A)
$$

In case of bei: ${ }^{\cdots} \mu \mathrm{F}$-continuous respect itself, we say simply that $\mu$ is $\mathrm{F}$-continuous.

The next is the main result in [1]:

Theorem 2.10. ([1]). If $\mu$ is $\mathrm{F}$-continuous respect to $\nu$ and $f_{n}, f \in \mathbb{E}(X)$ with $f_{n} \stackrel{\nu}{\longrightarrow} \int$, then $\int f_{n} d \mu \longrightarrow \int f d \mu$.

Rennark 2.11. We wish to point out that:

a) One of the more important aspects of this Theorem is that it proves the continuity of the fuzzy integral respect to measure convergence without using continuity conditions over tle fuzzy measure.

b) The authors consider fuzzy measures definite over $\mathcal{P}(X)$ because, essentially, if $\omega$ is an extension of $\mu$, then $\int f d \mu=\int f d \omega$.

\section{Autocontinuity and equivalences.}

We begin showing that the Theorem 2.10 is, in fact, an equivalence:

Theoren 3.1. Let $\mu, \nu$ fuzzy measures on $X$. Then, the following propositions are equivalents:

i) $f_{n} \stackrel{\nu}{\longrightarrow} f \Longrightarrow \int f_{n} d \mu \longrightarrow \int f d \mu$.

ii) $\mu$ is $\mathrm{F}$-continuous respect to $\nu$.

\section{Proof.}

ii) $\Rightarrow$ i) Theorem 2.10 .

i) $\Rightarrow$ ii) Suppose that $\nu\left(A_{n} \Delta A\right) \longrightarrow 0$.

It is clear that $\left\{x\left|\chi_{A_{n}}(x)-\chi_{A}(x)\right| \geq \mathcal{E}\right\} \subseteq A_{n} \Delta A, \forall n$.

Then, by monotony of $\nu$, we have that $\lim \nu\left\{x\left|\chi_{A_{n}}(x)-\chi_{A}(x)\right| \geq \mathcal{E}\right\}=0$.

But this means that $\chi_{A_{n}} \stackrel{\nu}{\longrightarrow} \chi_{A}$.

Thus, by liypothesis, we liave that $\int \chi_{A_{n}} d \mu \longrightarrow \int \chi_{A} d \mu$, wich implies that $\mu\left(A_{n}\right) \longrightarrow \mu(A)$ (see Remark 2.3). 
This proves that $\mu$ is $\mathrm{F}$-continuous respect to $\nu$.

As the definition of F-continuity involve, in general, more than one measure, we can think that it is a more extensive concept than the autocontinuity. Notwithstanding, they are equivalent in the sense:

Theorem 3.2. Let $(X, \mathcal{A}, \mu)$ a fuzzy measure space. Then:

$$
\mu \text { autocontinuous } \Longleftrightarrow \mu \text { is F-continuous respect to itself. }
$$
Jefinition:

Furthermore, we call prove a more general result which need the next

Definition 3.3. Let $\mu, \nu$ fuzzy measures over $X$ and $\mathbf{A}$ a $\sigma$-algebra of subsets of $\mathcal{A}$. We say that $\mu$ is autocontinuous with respect to $\nu$ over $\mathbf{A}$ if:

$$
A, A_{n} \in \mathbf{A} \text { and } \nu\left(A_{n}\right) \longrightarrow 0 \text { then } \mu\left(A \Delta A_{n}\right) \longrightarrow \mu(A)
$$

Theorem 3.4. Let $\mu, \nu$ fuzzy measures over $X$. Then:

$\mu$ autocontinuous respect to $\nu \Longleftrightarrow \mu \mathrm{F}$-continuous respect to $\nu$.

\section{Proof.}

$\Rightarrow$ Suppose that $\mu$ is autocontinuous respect to $\nu$ and let $\nu\left(A_{n} \Delta A\right) \longrightarrow 0$.

Then, by monotony of $\nu$, we have $\mu\left(A-A_{n}\right) \longrightarrow 0$ and $\mu\left(A_{n}-A\right) \longrightarrow 0$. Like $A \Delta\left(A_{n}-A\right)=A \cup A_{n}$ and $\nu\left(A_{n}-A\right) \longrightarrow 0$, then, by the autocontinuity of $\mu$ respect to $\nu$, we obtain:

$$
\mu\left[A \Delta\left(A_{n}-A\right)\right]=\mu\left(A \cup A_{n}\right) \longrightarrow \mu(A) .
$$

In the same way, like $A \Delta\left(A-A_{n}\right)=A \cap A_{n}$ and $\nu\left(A-A_{n}\right) \longrightarrow 0$ then

$$
\mu\left[A \Delta\left(A-A_{n}\right)\right]=\mu\left(A \cap A_{n}\right) \longrightarrow \mu(A) .
$$

Finally, using (3), (4) and the inequality $\mu\left(A \cap A_{n}\right) \leq \mu\left(A_{n}\right) \leq \mu\left(A \cup A_{n}\right)$, we conclude that $\mu\left(A_{n}\right) \longrightarrow \mu(A)$. Thus, $\mu$ is F-continuous respect to $\nu$.

$\Leftarrow)$ Suppose now that $\mu$ is $\mathrm{F}$-continuous respect to $\nu$ and let $\nu\left(A_{n}\right) \longrightarrow 0$. Using the identity $A \Delta\left(A \Delta A_{n}\right)=A_{n}$, we have $\nu\left[A \Delta\left(A \Delta A_{n}\right)\right]=\nu(A) \longrightarrow$ 0 . 
Then, by the $\mathrm{F}$-continuity of $\mu$ respect to $\nu$, we have $\mu\left(A \Delta A_{n}\right) \longrightarrow \mu(A)$. Consequently, $\mu$ is autocontinuous with respect to $\nu$.

In this context, the theorems 2.6 (Ralescu and Adams), 2.7 (Wang) and 2.10 (Greco and Bassanezi), are particular cases of the next more general result:

Theorem 3.5. Let $\mu, \nu$ fuzzy measures on $X, \mathbf{A} \subseteq \mathcal{P}(X) \sigma$-algebra. Then, are equivalent:

i) $\forall f_{n}, f$ A-measurables, $f_{n} \stackrel{\nu}{\longrightarrow} f \Longrightarrow \int f_{n} d \mu \longrightarrow \int f d \mu$.

ii) $\mu$ is autocontinuous with respect to $\nu$ on $\mathbf{A}$

Proof. Direct consequence of Theorems 3.1 and 3.4.

\section{Extensions and Autocontinuity}

Definition 4.1. Let $(X, \mathcal{A}, \mu),(X, \mathcal{B}, \omega)$ be fuzzy measure, spaces. We say that $\omega$ is an extension of $\boldsymbol{\mu}$ if $\mathcal{A} \subseteq \mathcal{B}$ and $\left.\omega\right|_{\mathcal{A}}=\mu$.

For example, if $\mathbf{A} \subseteq \mathcal{P}(X)$ is an $\sigma$-algebra of subsets and $\mu: \mathbf{A} \longrightarrow[0,1]$ is a fuzzy ineasure, then

$$
\begin{aligned}
& \mu^{*}(B)=\inf _{A \in \mathcal{A}}\{\mu(A) / B \subseteq A\} \\
& \mu_{*}(B)=\sup _{A \in \mathcal{A}}\{\mu(A) / B \subseteq A\}
\end{aligned}
$$

are extensions of $\mu$, respectively called outer and inner measures associated to $\mu$.

Theoren 4.2. Let $(X, \mathcal{A}, \mu)$ be a fuzzy measure space and let $\mu$ be autocontinuous witli respect to $v$ on $\mathbf{A}$. If $\omega$ is any extension of $\mu$ to $\mathcal{P}(X)$, then $\omega$ is autocontinuous with respect to $v^{*}$ on $\mathbf{A}$.

Proof. Let $A \in \mathcal{A}$ and $\left(B_{n}\right) \subseteq \mathcal{P}(X)$ be such that $v^{*}\left(B_{n}\right) \longrightarrow 0$.

By definition of $v^{*}$, given any $n \in \mathbb{N}$, we can find an $A_{n} \in \mathbf{A}$ such that $B_{n} \subseteq A_{n}$ and $0 \leq v\left(A_{n}\right)-v^{*}\left(B_{n}\right) \leq \frac{1}{n}$, which leads us to $v\left(A_{n}\right) \longrightarrow 0$. Consequently, we have

$$
v\left(A \cap A_{n}\right) \longrightarrow 0 \text { and } v\left(A_{n}-A\right) \longrightarrow 0
$$

Since $\mu$ is autocontinuous with respect to $v$ and $A \Delta\left(A \cap A_{n}\right)=A-A_{n}$ then, from (5), we obtain

$$
\lim _{n \rightarrow \infty} \mu\left[A \Delta\left(A \cap A_{n}\right)\right]=\lim _{n \rightarrow \infty} \mu\left(A-A_{n}\right)=\mu(A)
$$


Analogously, since $A \Delta\left(A-A_{n}\right)=A \cup A_{n}$ and $v\left(A-A_{n}\right) \longrightarrow 0$, then

$$
\lim _{n \rightarrow \infty} \mu\left(A \cup A_{n}\right)=\mu(A) .
$$

For other liand, $A-A_{n} \subseteq A \Delta B_{n} \subseteq A \cup A_{n}$ and $\left.\omega\right|_{\mathcal{A}}=\mu$.

So, making use of (6) and (7), it follows that

$$
\lim _{n \rightarrow \infty} \omega\left(A \Delta B_{n}\right)=\omega(A)
$$

From (8) We finally conclude this theorem.

Corollary 4.3. Let $\mu, v$ be fuzzy measures on $X$, then the following properties are equivalent:

i) $\mu$ is autocontinuous with respect to $v$ on $\mathbf{A}$,

ii) $\mu^{*}$ is autocontinuous with respect to $v^{*}$ on $\mathbf{A}$.

Corollary 4.4. Let $\mu$ be autoconitimuous with respect to $v$ oll $\mathbf{A}$ and $\omega$ be any extension of $\mu$ to $\mathcal{P}(X)$. If $f_{n} \stackrel{n^{*}}{\longrightarrow} f$ with $\left(f_{n}\right)$ and $f$ A-measurables, then $\int f_{n} d \omega \longrightarrow \int f d \omega$.

Corollary 4.5. With the same conditions of Corollary 4.4, then following properties are equivalent:

i) $\mu$ is autocontinuous with respect to $v$ on $\mathbf{A}$,

ii) $f_{n}, f$ A-measurables and $f_{n} \stackrel{v^{*}}{\longrightarrow} f \Longrightarrow \int f_{n} d \mu^{*} \longrightarrow \int f d \mu^{*}$.

\section{Some examples.}

Now, we give some examples which illustrate different aspects contained in this work.

Exauple 5.1. Let $X=\{a, b\}$ with $\mathcal{A}=\mathcal{P}(X)$ and let $\mu: \mathcal{A} \longrightarrow[0,1]$ be a fuzzy measure defined by

$$
\mu(A)=\left\{\begin{array}{lll}
1 & \text { if } & A=X \\
0 & \text { if } & A \neq X
\end{array}\right.
$$

If $A=X$ and $B_{n}=\{b\} \forall n$, then we liave $\lim _{n \rightarrow \infty} \mu\left(B_{n}\right)=0$ but $\lim _{n \rightarrow \infty} \mu\left(A \Delta B_{n}\right)=0 \neq \mu(A)$. Consequently, $\mu$ is not autocontinuous.

Let $f, g$ be functions such that : $f(a)=f(b)=1=g(a)$ and $g(b)=0$.

Define $f_{n}=f \forall n$. Observe that $f_{n} \stackrel{\mu}{\longrightarrow} g$ but $\lim _{n \rightarrow \infty} \int f_{n} d \mu=1 \neq 0=\int g d \mu$. 
In the next example we show that autocontinuity does not imply continuity.

Example 5.2. Let $\mu: \mathcal{P}(\mathbb{N}) \longrightarrow[0,1]$ be a fuzzy measure defined by

$$
\mu(A)= \begin{cases}0 & \text { if } A \text { is finite } \\ 1 & \text { if } A \text { is infinite. }\end{cases}
$$

We claim that $\mu$ is autocontinuous (with respect to itself). ln fact: Let $\left(A_{n}\right) \subseteq \mathcal{P}(\mathbb{N})$ with $\lim _{n \rightarrow \infty} \mu\left(A_{n}\right)=0$. Then, there is a number $n_{0}$ such that $\mu\left(A_{n}\right)=0, \forall n \geq n_{0}$. So, $A_{n}$ is finite for all $n \geq n_{0}$.

If $A \in \mathcal{P}(\mathbb{N})$, then we show that $\lim _{n \rightarrow \infty} \mu\left(A \Delta A_{n}\right)=\mu(A)$.

Case 1: $\mu(A)=0$.

In this case, $A$ is finite. Consequently, $A \Delta A_{n}$ is finite for all $n \geq n_{0}$.

Therefore $\mu\left(A \Delta A_{n}\right)=0 \quad \forall n \geq n_{0}$. In other words, $\lim _{n \rightarrow \infty} \mu\left(A \Delta A_{n}\right)=0=$ $\mu(A)$.

Case 2: $\mu(A)=1$.

In this case, $A$ is infinite. Hence $A \Delta A_{n}$ is infinite $\forall n \geq n_{0}$.

Therefore $\operatorname{lin}_{n \rightarrow \infty} \mu\left(A \Delta A_{n}\right)=1=\mu(A)$.

It follows that $\mu$ is autocontinuous. Put $A_{n}=\{1, \ldots, n\} \quad \forall n \in \mathbb{N}$ Then $\left(A_{n}\right)$ is monotone increasing with $\cup_{n=1}^{\infty} A_{n}=\mathbb{N}$ and $\mu\left(A_{n}\right)=0 \quad \forall n$. Hence $\lim _{n \rightarrow \infty} \mu\left(A_{n}\right)=0$ whereas $\mu\left(\lim _{n \rightarrow \infty} A_{n}\right)=\mu\left(\cup_{n=1}^{\infty} A_{n}\right)=\mu(\mathbb{N})=1$. Consequently, $\mu$ is not continuous.

Example 5.3. Let $(X, \mathcal{A}, \mu)$ be a fuzzy measure space. We say that $\mu$ is fuzzyadditive (f-additive) if : $A, B \in \mathcal{A} \Rightarrow \mu(A \cup B)=\max \{\mu(A), \mu(B)\}$. It's not dificult to see ([4]) that f-additivity implies autocontinuity. Thus, if $\mu$ is $\mathrm{f}$-additive then $f_{n} \stackrel{\mu}{\longrightarrow} f$ implies $\int f_{n} d \mu \longrightarrow \int f d \mu$. Observe that $\mu$, in example 5.2 , is $\mathrm{f}$ additive.

Example 5.4. Let $\mu$ be a fuzzy measure on $X$. We say that $\mu$ is a possibility measure if there exist a function $f: X \longrightarrow[0,1]$ such that $\sup \{f(x) / x \in X\}=1$ and $\mu(A)=\sup _{x \in A} f(x) \forall A \neq 0$ (with $\mu(0)=0$ ).

The function $f$ is called the density function associated to $\mu$. Clearly, if $\mu$ is a possibility measure then $\mu$ is f-additive. Consequently, the fuzzy integral is continuous with respect to "convergence in possibility".

Example 5.5. If $K \subseteq X$ then the "Dirac measure concertrated in $\mathrm{K}$ " is defined by:

$$
\mu_{K}(A)=\left\{\begin{array}{lll}
0 & \text { if } & A \cap K=0 \\
1 & \text { if } & A \cap K \neq 0
\end{array}\right.
$$


We observe that, if $\lambda K$ is a characteristic function of $K$, then we can write: $\mu_{K}(A)=\operatorname{sul}_{x \in A} \lambda K(x)$.

Hence, any cuncentrated (in K) Dirac measure is a possiblity neasure, with density function $f=\lambda \kappa$.

Example 5.6. Let $(\mathbb{N}, \mathcal{P}(\mathbb{N}), \mu)$ be as in example $52, \mathcal{A}_{\mathfrak{v}}=\left\{A \subseteq \mathbb{N} / A\right.$, $A^{c}$ is finite \}, and

$$
\mu_{0}(A)= \begin{cases}0 & \text { if } A \text { : finite } \\ 1 & \text { if } A \text { is tinite }\end{cases}
$$

Then $\mu$ is all extension of $\mu_{0}$. Moreover, $\mu=\mu_{0}^{*}$.

The fuzzy integral (fuzzy expectalion) can be interpreted in many ways. For instance, suppose that we evaiuate an object $\mathcal{O}$ from the point of view of a set of criteria $X$. Let $f(x)$ denote a grade of satistion that provides the object if criterion $x \in X$ is considered and let $\mu(\{;)$ denote the grade of importance of this criterion. Suppose now that we evdisiate the object taking into account criteria from the subset $A$ of $X$. It squing quite reasonable to consider a quantily $w(A)=\min _{x \in A} f(x ;$, as , bi best security grade of satisfaction i...it.

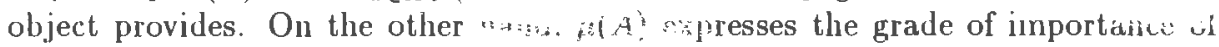
this subset of criteria. comparing ace a quantities (in the sense of $\wedge$ operator) we obtain a quantity that may in merpreted as the grade of agreement between real possibilities (expressed in terms of $f(x)$ ) on the one hand, and our expectations (expressed in terms of $\mu$ ) c: the other one. Hence the fuzzy integration may be interpreted as a searching for maximal grade of agreement between two opposite tendencies (actuaily, if $A \subseteq B$ then $\mu(A) \leq \mu(B)$ whereas $w(A) \geq w(B)$. See [1] alld [5].

\section{REFERENCES}

[1] Greco, F. Bassanezi, R.C.: On the continuity of fuzzy integrals. Fuzzy Sets and Systems, $53 \mathrm{~N}^{\circ} 1$ (1993), 87-91.

[2] Greco, G.; Bassanezı, R.C.: Continuidade das fuzzy integrais. Atas a $\mathfrak{z}^{\infty}$ Sem. Bras. Anal Ra de Janeirc. 1995.

[3] Ralescu, D.; Adams, G. The Luzg :atcgrais. J. Anal Math. Appl, 75 (1990), 562-570.

[4] Román-Flores, H.: Sobre entropías fuzzy. Ph.D.Dissertation, IMECCUNICAMP, Campınas-SP, Brasil, 1989.

[5] Sugeno, M.: Fuzzy measures and fuzzy integrals: A survey, in: M. Gupta et al., Eds. Fuzzy Automata and Decısıon Process (North-Holland, Amsterdam, (1977), 89-102. 
[6] Wang, Z.: The autocontinuity of set function and the fuzzy integral. $J$. Math. Anal. Appl., 99 (1984), 195-218.

Received: July 2, 1993

Arturo Flores F. and Heriberto Román F.

Departamento de Matemática

Facultad de Ciencias

Universidad de Tarapacá

Casilla 7-D, Arica, Chile.

Rodney C. Bassanezi

IMECC

Universidad de Campinas

CP 6065 13081-970-Campinas-SP, Brasil. 